\title{
Research and Application of Production Material Management System in Forging Center Based on Workflow and Rule Engine
}

\author{
LI Yuejun ${ }^{1, a}$ \\ ${ }^{1}$ College of Optical and Electronical Information, Changchun University of Science and Technology, \\ Changchun, China \\ ayue_jun15@163.com
}

Keywords: forging center; production material management system; business rule engine; workflow

\begin{abstract}
Different kinds and types of forge pieces in forging center in Railway Vehicle Company lead to different management process of large amount of production materials, which introducing different workflow, resulting in heavy workflow engine load and huge workload for defining workflows. To address this problem, rule engine was combined with workflow technique to simplify workflow number and improve system flexibility to changes, thus prolonging system lifetime and greatly improving system efficiency. Based on the proposed method, the function structure design on forging center Production Material Management System (PMMS) was provided to lay solid foundation for design and implement of information management system.
\end{abstract}

\section{Introduction}

Some railway vehicle company owns leading infrastructure, craft equipment as well as $R \& D$ capabilities and level in the aspects of forge, cast, machine-made, rivet weld and spinning, which mainly produces vehicle axles, forges frame of trail hook and spins brake cylinder. As to railway vehicles, the forging pieces of each type are tens of thousands. The materials consumption amount is large. The production materials involve different management processes and users. Therefore, it needs to integrate management on production materials in the forging center PMMS. The production material management includes activities involved with whole production starting from storage of raw materials of enterprises, ending with finished products storage based on regulated process for processing and storage in accordance with product form. Production materials management researched in the paper mainly focuses on logistics in the workshop production process. Workshop logistics refers to production material flow within or between workshop, including the process from material been received into workshop from warehouse, production within workshop, material transfer between workshops as well as production into warehouse. There is thermal processing in the forging production process. Meanwhile, strict quality investigation should be accomplished after process finished. Therefore, the workshop takes process verification result as material distribution and transformation. In the process of forging center, there production material is often shortage. It needs to obtain storage information in the task customization. In case of shortage, it should be treated timely. In order to ensure normal production without interruption, the job plan should ensure material availability and surplus of working capacity. According to above analysis, it can be seen that the difficulty is using which technique to represent collaboration among users into an organic whole. In addition, different production material introduces large amount of different management process. It needs to determine how to combine and simplify management processes. Furthermore, the system should also response to changes of management process caused by future production user or material adjustment, which has not been involved in existing PMMS of forging center or forging workshops. Huang et al. aimed at intelligent forging process design to integrate forging expert system, process card draw system, information management system and process information statistical information based on database [1]. Yu et al. constructed production information model of forging process based on integrated CAPP Framework and development platform [2]. The knowledge base and rule based were also built. Mathematical methods of model decision and logic decision were used to implement 
process decision to build forging CAPP system. Li et al. established a heating treatment workshop management system with object-oriented, component-based software reuse and web techniques [3]. The system structure and functions were analyzed and key model technologies were analyzed. Ran $e t$ al. developed and designed forging workshop management information system based on computer production process monitoring and management system in accordance with ITIL theory so that existing information resource to play greater efficiency [4]. He et al. developed tool and mold management system in forging workshop to solve human source waste [5]. The above researches proposed requirements on forging workshop management information, but function is sole. Some are simple management on parts of forging workshop, or functional module partition on workshop management, which lacks of detail researches on whole forging production workshop, especially to important job scheduling, material management and etc. Starting from production material management, the method to manage production materials with rule engine combining with workflow technique was discussed. The functional structure design of material management system was also given. The paper is organized as follows: section 2 gives production material management process based on workflow; section 3 provides simplification method based on rule engine; section 4 gives specific application on forging center information management; section 5 concludes our work.

\section{Production Material Management Process Based on Workflow}

Workflow Technology. Workflow management system (WfMS) mainly completes partly or totally automatic of business process. It transfers needed documents, information or tasks from on engaged activity node to next engage one in accordance with certain process rule set [6]. It describes whole business process into process assembled by a series of activities. The whole system can be abstracted as system $S(A, U, F, M, E)$. Where, $A$ is the set of a set of activities on documents, information or tasks; $U$ is user set that completes responding activities in the system; $F$ is workflow set and each flow $F_{X}$ is an ordered sequence made up of activity $\left\{A_{x}, \cdots, \mid A_{x} \in A\right\} ; M$ is flow customizer to map $A$ to $F$, which can express existing business process into workflow of $F_{x}$; $E$ is workflow engine, which explains execution on flow in $F$ driven by events. The operation representation of a typical workflow is shown in Fig. 1.

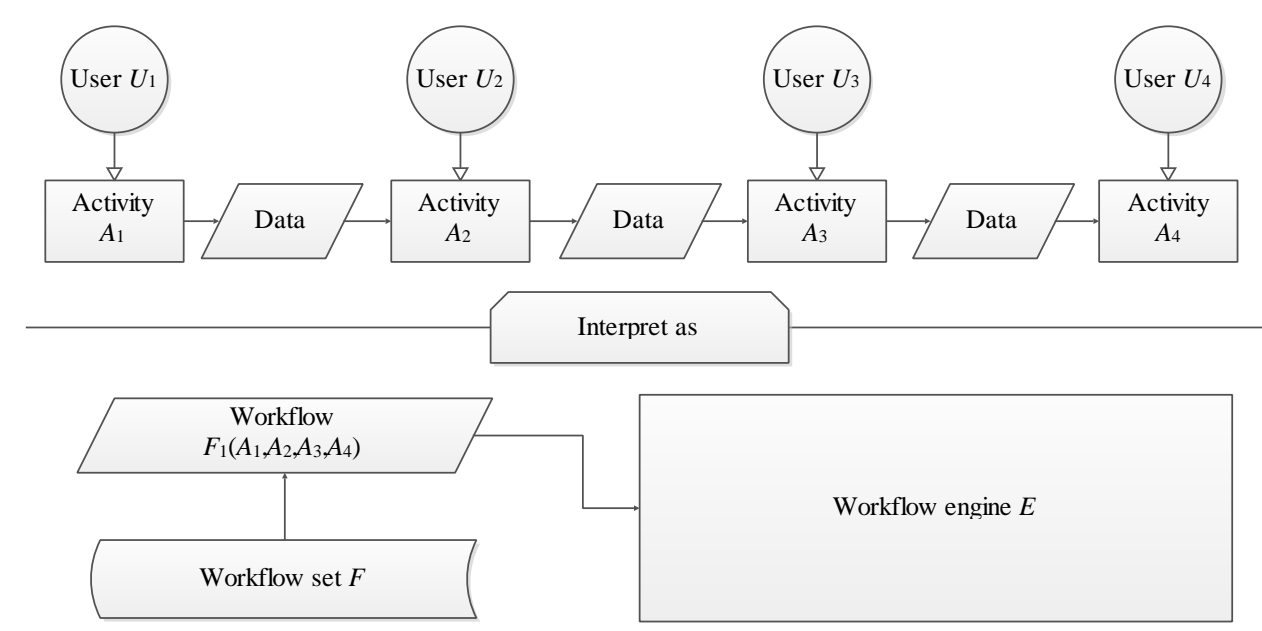

Figure 1. Typical workflow representation.

Production Material Management Process Modeling. We can see from the comparison between forging center PMMS and workflow management system that both have two aspects for analogy. One is business management process in PMMS is actually information flow related to production material in different business activities, while data flow among different activities in workflow management system. Another is that user execute corresponding activities in PMMS to process received information, while is similar to data processing on corresponding activities in the workflow management system. In other words, business process, user and business information 
processing in the PMMS can be represented by flow, user and activity in workflow management system.

Based on above analysis, the PMMS can be abstracted into an example $\operatorname{IS}(A, U, F, M, E)$ of workflow management system. Where, $A$ is defined as $\left\{A_{x}\right\}$ to denote activities on production material as information collection, plan application, aggregation, material into plant, transfer as well as test. The $U$ is set of various users in the system, which completes activities on material information on different terminals. The $F$ is set of business flow, which is the member order set of $A$. The $M$ is business flow definer, which maps activities defined by $A$ in business flow into examples of $F$ in accordance with different material class and type. The $E$ is a specific example of workflow engine in above workflow management system. Fig. 2 shows a business flow of whole system from application to approval activities.

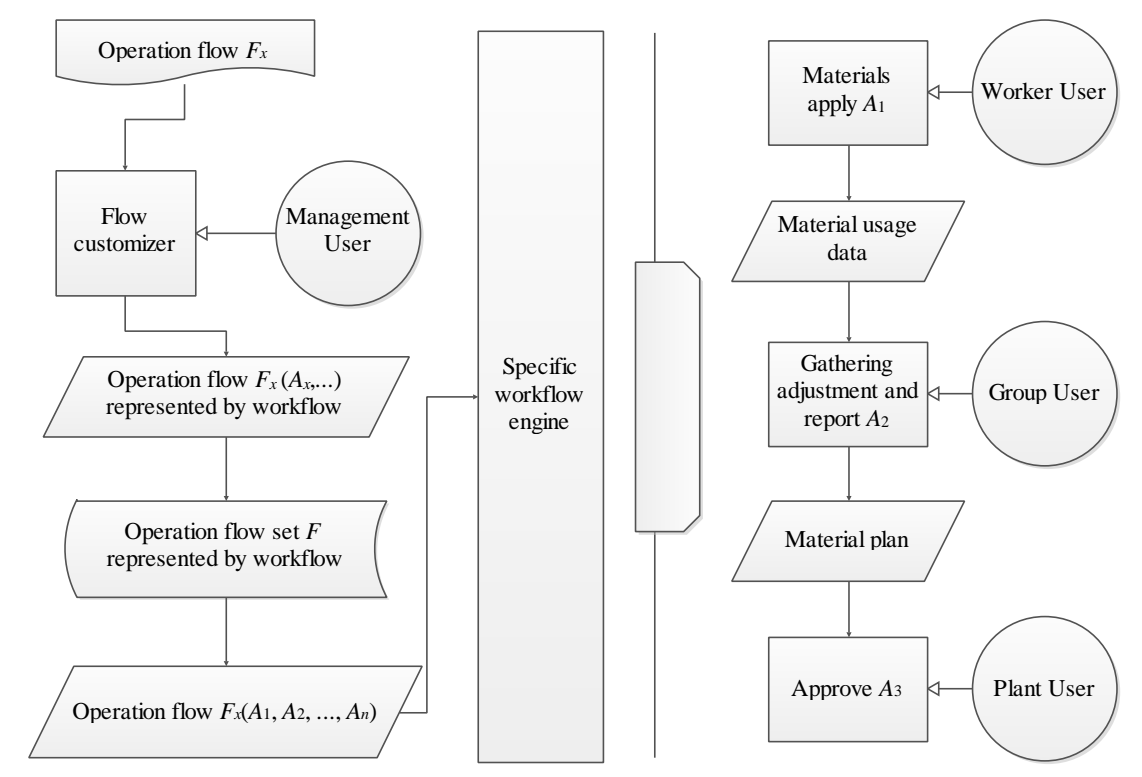

Figure 2. Production material management business flow example.

Using workflow management system can better describe the PMMS, but it also has two serious problems. Firstly, the system should customize different workflow according to different classes and types of material, so the amount of workflow is too much, resulting in heavy burden of workflow engine and the work to define workflow is huge. Furthermore, in case of new type of material been introduced into PMMS, new workflow should be defined, thus the maintenance is also large. Secondly, active nodes in the system is closely to future changes or is lowly automation, so majority should be manually processed. For example, different material has different quality evaluation standard. If the evaluation algorithm automatically completes encoding in active node, it cannot deal with new material type without changing coding. If it is changed into manually processing, the automation is lower. In order to address these problems, other techniques should be introduced to simplify workflow number and improve system strain capacity to changes.

\section{Flow Simplification Based on Rule Flow Engine}

We can see from the above analysis that the reason resulting in too many workflow is system needs to define workflow according to material classes and types independently. Obviously, there are many similar workflows in the system. After eliminating some nodes, some flow can be changed into another one. For example, there are flows $F_{1}\left(A_{1}, A_{3}, A_{5}, A_{7}, A_{8}\right), F_{2}\left(A_{1}, A_{3}, A_{7}, A_{8}\right)$ and $F_{3}\left(A_{1}, A_{7}, A_{8}\right)$. Although $F_{1}$ has one more activity $A_{3}$ compared with $F_{2}$, the relative sequence of them are same. Statistics shows that the flow number will decrease more than $95 \%$ if activities similar to $F_{1}, F_{2}$ and $F_{3}$ come down to $F_{1}$. In order to implement this goal, it requires active node has some intelligence so that it can ignore materials need not processing. Obviously, it is not appropriate to achieve this with 
hard encoding method in that it enables flow changes or addition of new material to be impossible. Combining with needs to add robustness of active node, the paper introduces a technique to enhance intelligence of activity without decrease ability to deal with changes.

The paper introduces business rule engine to solve the problem. It is an component embedded into application, the task of which is to test and compare data objects submitted to engine currently to business rules loaded in the engine and written in rule language, so as to activate business rules fitting to current data status. According to rule language logic, it can modify data object or trigger corresponding application operations, including functional modules as matcher, executor, working memory, rule base and etc. [7]. The working principle is shown in Fig. 3.

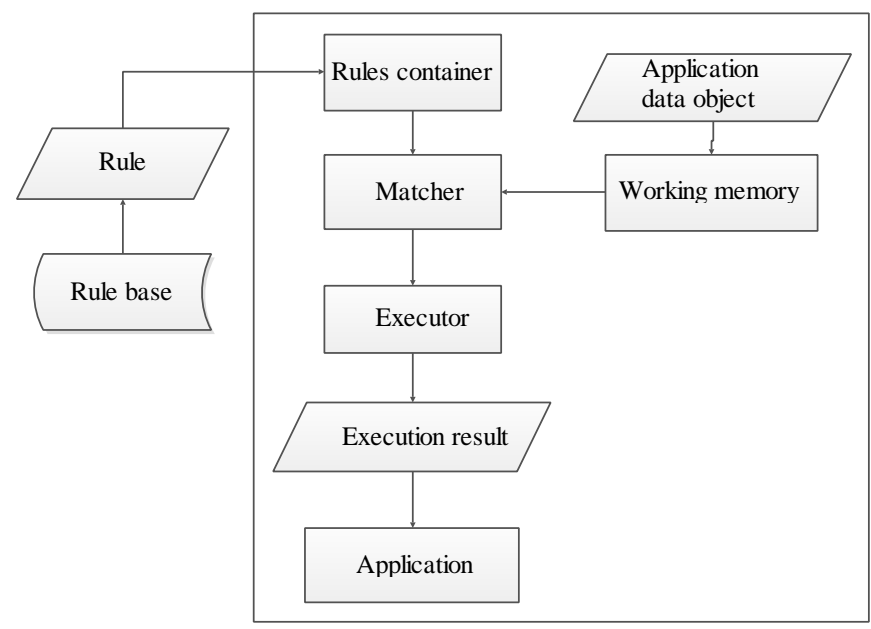

Figure 3. Rule engine structure.

The rule engine is used in active nodes in the PMMS to introduce necessary intelligence of system, thus soling above two problems. We can write rule language code similar to following to ship processing material types or adding ability to evaluate material quality automatically.

if Material. Type Equal 'Steel' then

Sendto Next Activity

end if

if Material.Weight Exceeds 10000 then

NextActivity='Storage'

end if

When the new material added or management flow and information process algorithm changed, we only need to modify above rule code, thus greatly enhanced the ability of the system to deal with change.

\section{Forging Center Information Management System Application}

Based on above research result, .NET Workflow framework and ILog Rule Engine for .NET are used to implement PMMS. The system uses .NET WF to organize business flow and deploys ILog Rule Engine at active nodes to enhance system robustness. The function structure of whole system is shown in Fig. 4. It includes five parts, namely basic data, business activities, flow customization, transaction to do as well as system settings and maintenance. Basic data is used to maintain all production material and users in the system. With these basic data, the system generates business object to be transferred in the workflow. When these business objects transferred to active nodes, they are firstly processed by rule engine and then return to other parts in the system. Business object includes basic business processing parts as production material information collection, usage plan application, summary, application, approval, task deployment, material into plant, transfer, test and etc. These activities are processed by business objects after rule engine processing with human-machine interface. After each time of processing, it only needs to perform next one. Business 
flow customization is executed by system administrator with activities in the workflow in accordance with business flow. Meanwhile, the business object to be transferred in the workflow should be specified. If desired, the rule code on each active node can also be edited. These rule code can only be operated by specific objects by the workflow or to execute specific application operations. Transaction to do function mainly provides workflow tasks all flows into this node. The system settings and maintenance function mainly includes data backup and recovery, permission set, encryption set and maintenance functions.

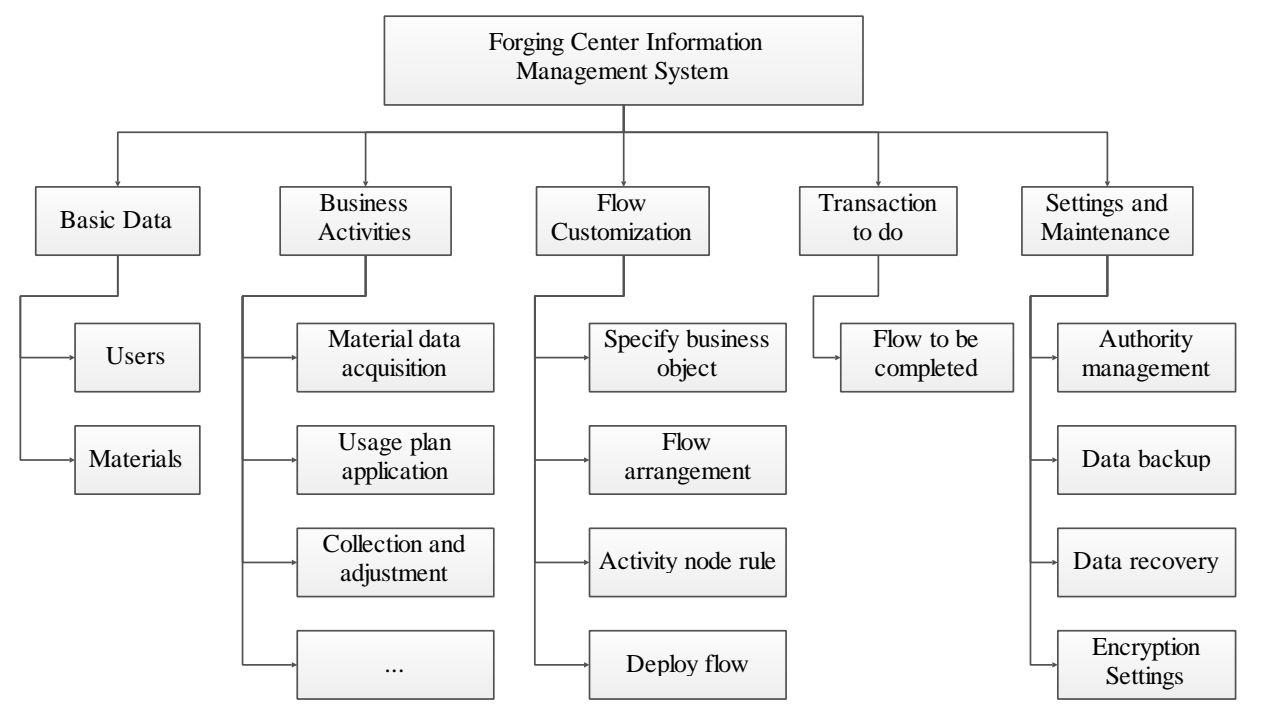

Figure 4. Function structure of forging center information management system.

\section{Conclusion}

The combination of workflow and rule engine provides ideal technique platform for implement of PMMS. Compared with traditional workflow technique system, it reduces workflow number, thus decreasing work to define workflow and greatly reducing workload for system maintenance. More importantly, it improves system ability to deal with changes, thus prolonging system lifetime and greatly improving system efficiency and investment ratio. Meanwhile, this technique is also found some problems, such as management flow modification change should also consider changes of corresponding rule code, which improves requirements on capability of system maintenance personnel. In the next future, we will start from more intelligent rule engine to design and implement perfect production material management system.

\section{References}

[1] Huang Y., Zhu Y. L., and Li D. B., Development of the Forging CAPP Expert System, Mechanical Engineer, no. 10 (2006) pp. 61-63.

[2] Yu X. H., Dong Q. Y., and Yang G. L., A CAPP system of forging process based on CAPP Framework, Metalforming Machinery, no. 10 (2001) pp. 61-63.

[3] Li H., Xie Q. S., and Lin W., Study and application of management system for heating treatment workshop, Journal of Guizhou University of Technology, vol. 34, no. 5 (2005) pp. 51-54.

[4] Ran C. S., and Zhao P., The development and application of forging plant information management system, Heat Treatment Technology and Equipment, vol. 27, no. 3 (2006) pp. 62-64.

[5] He D. J., and Zhou C. H., Tool and mold management system in forging workshop, Metalforming Machinery, no. 5 (2001) pp. 52-53. 
[6] Brockman J. B., and Director S. W., The schema-based approach to workflow management, IEEE Transactions on Computer-Aided Design of Integrated Circuits and Systems, vol.14, no. 10 (1995) pp. 1257-1267.

[7] Maclintock C., and Eberling W., ILog JRules technical white paper, 2002.

[8] Li C. F., and Tan Q. P., Business Rule-based Workflow Model, Computer Engineering and Applications, vol. 42, no. 26 (2006) pp. 65-67. 